\title{
$\widehat{A}$ Madridge \\ madridge Journal of Nursing \\ interconnecting Scientific world
}

Research Article

Open Access

\section{Nurses' Perceptions of Incivility in the Operating Room}

\author{
Katy Garth, Tonia Mailow, Nancy Armstrong*, Dana Todd and Dina Byers \\ School of Nursing \& Health Professions, Murray State University, Murray, Kentucky, USA
}

\section{Article Info}

\author{
*Corresponding author: \\ Nancy Armstrong \\ School of Nursing \& Health Professions \\ Murray State University \\ Kentucky \\ USA \\ Tel: 2708094576 \\ E-mail: narmstrong1@murraystate.edu
}

Received: November 18, 2019

Accepted: November 28, 2019

Published: December 05, 2019

Citation: Garth K, Mailow T, Armstrong N, Todd D, Byers D. Nurses' Perceptions of Incivility in the Operating Room. Madridge J Nurs. 2019; 4(1): 164-166.

doi: 10.18689/mjn-1000129

Copyright: ( 2019 The Author(s). This work is licensed under a Creative Commons Attribution 4.0 International License, which permits unrestricted use, distribution, and reproduction in any medium, provided the original work is properly cited.

Published by Madridge Publishers

\begin{abstract}
The operating room (OR) can be a high-stress area for healthcare workers. A great deal of expertise, teamwork, and precision is needed for the area to run smoothly and achieve desired patient outcomes. Incivility in healthcare has been recognized in the literature as a cause of healthcare worker distraction with increased risk of medical errors, reduce collegiality, and employee turnover. A survey was sent to nurses who work in operating rooms in a southern state. Forty-three nurses responded to the survey. It was found that most had experienced incivility in the workplace (85\%). Fiftyeight percent stated that they had dreaded going to work due to workplace incivility. Twenty-eight percent said that they had experienced stress-related illnesses due to workplace incivility. Physicians and fellow nurses were found to be the most common perpetrators of uncivil behaviors in the $O R$, compared with supervisors, patients, or other hospital employees. The study's findings underscored the need for hospitals and healthcare systems to focus on reducing incivility the operating room.
\end{abstract}

Keywords: Workplace Incivility; Lateral Violence; Bullying; Operating Room; Nursing.

Abbreviations: OR: Operating Room; NIS: Nursing Incivility Scale; IRB: Institutional Review Board.

\section{Introduction}

The need to reduce workplace incivility in healthcare settings has become an area of increasing concern. Workplace incivility is defined as, "acting rudely or discourteously, without regard for others, in violation of norms for respect in social interactions" [1]. Incivility in the workplace can include behaviors that are overt, such as verbal outbursts or rudeness, or covert, including gossip or backbiting. It can also include exclusionary behaviors, such as not providing needed information for patient care or information needed for effective job performance. Workplace incivility may affect the ability of healthcare workers to provide safe, effective care of patients. Nursing workplace incivility has been found to increase the risk of errors, lower morale and job satisfaction, and increase job turnover $[2,3]$.

The operating room (OR) can be particularly prone to workplace incivility, due to its high-stress, intricate nature. Operating rooms in the United States and Canada have been found to be affected by uncivil behaviors. In a recent survey of 7,465 operating room staff in the United States (US) and Canada, 667 staff members stated that they had experienced abuse on a personal level and 1,121 clinicians had witnessed the abuse of colleagues [4]. An international survey of 7,465 operating room workers found that $98 \%$ were exposed to some form of disruptive behavior in the surgical setting, with an estimated average of 64 exposures to operating room disruptions per year [5]. Professional organizations are recognizing the need to address workplace incivility in the OR. The American Association of Nurse Anesthetists (AANA) have developed an accreditation standard focused on 
incivility in the OR, Promoting a Culture of Safety and a Healthy Work Environment [6]. The standard addresses reducing workplace incivility and creating a culture that encourages a supportive environment in the operating room and promotes the safety and health of patients, as well as operating room staff. There is limited data available focusing specifically on incivility in the OR setting. Given the potential impact of incivility in the $O R$, the researchers sought to explore the perception of incivility among nurses working in operating rooms, this time in primarily rural settings in one US state.

\section{Methods}

The purpose of this study was to examine operating room nurses' perceptions of the impact of workplace incivility in the $\mathrm{OR}$. The researchers compiled a three-part survey that included the Nursing Incivility Scale (NIS), questions about personal experiences with incivility in the workplace, and demographic data. The Nursing Incivility Scale (NIS) is a tool with established validity and reliability that uses a Likert-type scale to measure incivility in the workplace [7]. Additional questions were included that addressed personal experiences with workplace incivility, the consequences of exposures to incivility in the OR, and the response of hospital leaders and management to reported occurrences of incivility in the OR. This section of the survey incorporated a mixture of question types, including yesno and multiple-choice items. If the participant answered "yes" about exposure to uncivil behaviors, additional questions were asked to clarify their reaction or actions taken following the event. Some questions used a seven-point Likert-type scale with responses ranging from strongly disagree (1) to strongly agree (7). For example, one question asked if the OR nurse had dreaded going to work because of uncivil behavior with responses on the seven-point scale. Demographic data, such as age and years of experience in the OR were also collected.

The researcher developed a plan to make the survey available to active $O R$ nurses throughout one southern state. A list of all hospitals in the state was obtained. At least one attempt was made to contact the chief nurse administrator or operating room director at each hospital or hospital system that had an OR department to ask for permission to share the survey via email with the OR nurses at the hospital. When permission was granted, the nurse leader was then asked to forward an email to the OR nurses that included a link to the electronic survey. An explanation of the purpose of the study was included with the email, along with a statement explaining that that survey responses would be sent to the researchers only and only in the aggregate. Individual survey results would remain confidential and anonymous. Participation was voluntary and completion of the survey implied consent. Prior to implementation of the study, institutional review board (IRB) approval was sought and the study was found to be exempt from the need for oversight (IRB \#17-104).

\section{Results}

Forty-three OR nurses returned completed surveys. The majority of participants, $86.05 \%(n=37)$, were female, $13.95 \%$ $(n=6)$ were male. There was little ethnic diversity in the sample with $95.35 \%(n=41)$ reporting that they were Caucasian. Ages ranged from 30 to over 59 years. The $40-49$ year block comprised the largest group, $41.86 \%(n=18)$, followed by $30-39$ year olds, $25.58 \%(n=11)$, and $50-59$ year olds, $25.58 \%(N=11)$, three participants were over 59 years of age. Over one third of the nurses responding to the survey had been employed as a nurse for over 20 years $(37.5 \%, n=15)$ and reported they had more than 20 years of experience $(28.57 \%, n=12)$ in the OR. The majority of the participants practiced in communities with populations less than $100,000(74.42 \%, n=32)$, with almost half of participants working in communities with populations under 50,000 .

A majority of the participants, reported that they had experienced incivility in the workplace, with $85.71 \%(n=36)$ indicating that they somewhat agreed, agreed, or strongly agreed. Participants were asked if they had reported uncivil workplace behavior in the OR to a supervisor or other administrative personnel. Those responding "yes" accounted for $58.54 \%(n=24)$ of the responses. Next, the participants who responded "yes" were asked if their report was taken seriously, with $34.79 \%(n=8)$ reporting that they strongly disagreed or disagreed.

The participants were asked if they had developed a stressrelated illness due to incivility at their workplace. Responses of agree or strongly agree were selected by $28.09 \%(n=11)$ of the participants. Fifty-six percent $(n=22)$ of the participants stated that they somewhat agreed, agreed, or strongly agreed that they have dreaded going to work due to uncivil behavior in the operating room. Uncivil behaviors were reported most commonly as being committed by fellow nurses or physicians in the OR. The participants reported little uncivil behavior from supervisors, patients, or other hospital employees. The most common uncivil behavior that the participants reported as being perpetrated by fellow OR nurses was gossiping about other nurses, with $83.79 \%(n=31)$ reporting. Sixty-six percent $(n=23)$ of the OR nurses reported some form of uncivil behavior, such as taking out stress or anger on others, from physicians toward OR nurses or staff, with responses of agree or strongly agree. Despite these troubling behaviors, most $(64.05 \%, n=25)$ of the participants believed that there is at least some effort at their workplace to create a culture of civility.

\section{Discussion}

The perception and experiences of incivility experienced by the OR nurses in this study are consistent with those reported in other studies, underlining the pervasiveness of incivility in healthcare. Most of the participants in this study were exposed to incivility in the operating room at some point in their career. Most commonly, physicians and fellow nurses were the perpetrators of uncivil behaviors in the OR. Gossiping by fellow nurses and lashing out behaviors by physicians were the most commonly reported uncivil behaviors experienced by operating room nurses. Many of the nurses in this study reported that they had become physically ill or dreaded coming to work, due to an uncivil workplace. A majority of the nurses who experienced or observed uncivil behavior in others had 
reported it to a supervisor. However, around one-third of the participants felt that their report was not taken seriously. Despite these outcomes, many of the OR nurses participating in the study reported that they recognized that their hospital and surgical unit was trying to promote a civil work environment.

When uncivil behavior is allowed to occur in operating rooms, the potential consequences can affect patient safety. In a recent a randomized controlled trial, a group of researchers created a patient simulation involving a crisis occurring in the $\mathrm{OR}$, during a procedure [8]. Half of the simulations involved a normal work environment, where the other half allowed uncivil behaviors to occur during the simulated surgical crisis. All performance metrics, such as vigilance and patient management, were lower in the group in which the participants were exposed to rude behavior. Over half (65\%) of the participants in the experimental group felt that their ability to perform during the crisis was affected by the exposure to incivility.

A dissertation focusing on incivility in the operating room found a correlation between nurses' intent to leave a position and job satisfaction with disruptive behaviors in the operating room [9]. Surveys were sent to OR nurse in five hospitals in Maryland with 51 participants responding to the survey measuring stress levels, job satisfaction, and exposure to disruptive behaviors. Intent to leave had a moderately positive correlation with exposures to disruptive behavior in the operating room. Job satisfaction correlated negatively with disruptive behavior in the operating room. Disruptive behaviors in the OR can result in reduced job satisfaction, which can in turn lead to nursing turnover, disruptions in care continuity, and economic impacts on the organization, as the unit must replace nurses who leave due to uncivil work environments.

Villafranca, Fast, and Jacobsohn have focused most of their recent research on incivility in the OR. They recommend that professional standards and codes of conduct include the maintenance of civil work environments in operating rooms, and that clinicians be held to those standards, with transgressions addressed as they occur [5]. Open communication should be a hallmark of the management styles of OR managers and leaders, so that behavior can be reported, fully investigated, and addressed without fear of retribution or the automatic acceptance of reports of uncivil behaviors without a full investigation. The process should be open and collaborative.

Clark, one of the foremost experts on nursing workplace incivility, recommends that OR incivility be addressed by nurse managers and leaders by creating an environment that promotes teamwork, and that they personally model civil behavior in the workplace [10]. Clark encouraged surgical units to develop policies that address uncivil or disruptive behaviors in the operating room and that those policies truly be enforced in the workplace. Training programs that focus on the ability to recognize and effectively respond to workplace incivility as it occurs can also be helpful to all members of the OR. Cognitive rehearsal, where effective responses to workplace incivility are practiced mentally prior to exposure, has been found to be an effective tool in training nurses to successfully and confidently respond to uncivil behaviors in the workplace $[10,11]$.

\section{Conclusions}

Incivility can have a negative impact on healthcare workers. Before the problem can be addressed, nurses must recognize behaviors that are considered uncivil in themselves and others. Nurses should demonstrate or model appropriate behavior in the workplace setting. If uncivil behavior is witnessed or experienced, it should be reported. At the same time, organizational leadership should take the report seriously. It is the responsibility of hospital administrators and supervisors to foster open communication about uncivil behaviors. If reported uncivil behavior is not taken seriously, nurses may be less likely to report incivility in the future. Healthcare organizations must implement clear policies that support "zero tolerance" for incivility. If incivility is to be overcome, healthcare workers must demonstrate and facilitate positive working relationships among each other.

\section{Conflicts of Interest Statement}

The authors declare no competing interests.

\section{References}

1. Andersson LM, Pearson, CM. Tit for Tat? The Spiraling Effect of Incivility in the Workplace. The Academy of Management Review. 1999; 24(3): 452471. doi: $10.2307 / 259136$

2. Wright $\mathrm{W}$, Khatri N. Bullying among nursing staff: Relationship with psychological/behavioral responses of nurses and medical errors. Health Care Manage Rev. 2015; 40(2): 139-147. doi: 10.1097/ HMR.00000000000000015

3. Rahim A, Cosby DM. A model of workplace incivility, job burnout, turnover intentions, and job performance. Journal of Management Development. 2016; 35(10): 1255-1265. doi: 10.1108/JMD-09-2015-0138

4. Villafranca A, Magid K, Young A, Fast I, Jacobsohn E. Abusive behaviour in Canadian and US operating rooms. Can J Anaesth. 2019; 66(7): 795802. doi: 10.1007/s12630-019-01334-7

5. Villafranca A, Fast I, Jacobsohn E. Disruptive behavior in the operating room: prevalence, consequences, prevention, and management. Curr Opin Anaesthesiol. 2018; 31(3): 366-374. doi: 10.1097/ ACO.0000000000000592

6. AANA. Promoting a culture of safety and healthy work environment practice considerations. American Association of Nurse Anesthetists. 2015.

7. Guidroz AM, Burnfield-Geimer JL, Clark O, Schwetschenau HM, Jex SM. The nursing incivility scale: development and validation of an occupation-specific measure. J Nurs Meas. 2010; 18(3): 176-200. doi: 10.1891/1061-3749.18.3.176

8. Katz $D$, Blasius $K$, Isaak $R$, et al. Exposure to incivility hinders clinical performance in a simulated operative crisis. BMJ Qual Saf. 2019; 28(9): 750-757. doi: 10.1136/bmjqs-2019-009598

9. Reyka ME. The Impact of Disruptive Behavior on Operating Room Nurse Satisfaction. Theses and Dissertations (All). 2015; 508.

10. Clark CM, Kenski D. Promoting Civility in the OR: An Ethical Imperative. AORN J. 2017; 105(1): 60-66. doi: 10.1016/j.aorn.2016.10.019

11. Armstrong N. Management of Nursing Workplace Incivility in the Health Care Settings: A Systematic Review. Workplace Health Saf. 2018, 66(8): 403-410. doi: 10.1177/2165079918771106 